\section{Temporal lobe volume changes in people at high risk of schizophrenia with psychotic symptoms}

\author{
STEPHEN M. LAWRIE, HEATHER C. WHALLEY, SUHEIB S. ABUKMEIL, \\ JULIA N. KESTELMAN, PATRICK MILLER, JONATHAN J. K. BEST, \\ DAVID G. C. OWENS and EVE C. JOHNSTONE
}

Structural brain imaging studies in schizophrenia have demonstrated several abnormalities, particularly of the temporal lobes and constituent parts, both in people with established disease and in first-episode patients (Suddath et al, 1990; Shenton et al, 1992; Lawrie \& Abukmeil, 1998; Lawrie et al, 1999; Wright et al, 2000). This has generally been interpreted as indicating that the brain changes are present early in life, but the timing is unknown. The Edinburgh High-Risk Study was designed to address this uncertainty. Participants were recruited as adolescents, having at least two affected relatives. Structural magnetic resonance imaging (MRI) of the brain at baseline revealed that the mean volumes of the amygdala-hippocampus complex in the brains of people at high risk were smaller than those of healthy participants but larger than in first-episode patients (Lawrie et al, 1999, 2001). We scanned subgroups of high-risk and healthy control participants on two occasions about 2 years apart. Some of the high-risk participants had experienced psychotic symptoms during this time, several at baseline assessment. We therefore test two hypotheses in this report. First, we determined whether people at high risk generally show a reduction in the volumes of the temporal lobes and amygdalahippocampus over time compared with a healthy control group. Second, we examined whether these structures reduced in volume in high-risk participants with psychotic symptoms.

\section{METHOD}

Ethical approval was obtained from all relevant health boards and enrolment began in 1995. After a complete description of the study had been given to participants, their written informed consent was obtained.

\section{Participants}

Details of the recruitment process have been described in previous papers (Hodges et al, 1999; Johnstone et al, 2000). Briefly, individuals with schizophrenia, with a family history of schizophrenia and with adolescent relatives, were identified from psychiatric hospital case records in most areas of Scotland. Case-note diagnoses of schizophrenia were verified with the Operational Criteria Checklist (McGuffin et al, 1991). We then approached their relatives, and high-risk subjects aged 1625 who agreed to participate were given a detailed clinical, neuropsychological and brain imaging assessment. The clinical assessment included the Present State Examination (PSE, ninth edition), a structured psychiatric interview schedule which has been widely used in studies of schizophrenia (Wing et al, 1974). All these assessments were repeated after approximately 2 years in consenting participants who had enrolled in the first 2 years of the study.

A control group of healthy individuals without any family history of schizophrenia was recruited from the same areas of Scotland as the high-risk participants. These people were of similar age to the high-risk group and their numbers were comparable to the number of people expected to develop psychosis. Members of the control group were fully examined as above and consenting individuals reassessed after approximately 2 years.

\section{Symptom measures}

At each clinical assessment, psychotic and other symptoms were identified and measured on a videotaped PSE. Participants were initially divided into five groups on the basis of symptom severity (Johnstone et al, 2000):

\section{0 - no symptom \\ 1 - any fully rated non-psychotic symptom or behavioural item}

2 - partially rated psychotic symptoms (mainly isolated perceptual disturbances or partially held delusions) or behaviours

3 - any fully rated psychotic symptoms (hallucinations and delusions) or behaviour

4 - schizophrenia diagnosed according to PSE and ICD-10 (World Health Organization, 1992) criteria.

For statistical analysis groups rated 0/1 and 2/3 were collapsed (absence or presence of psychotic symptoms), and those who developed schizophrenia before the second scan could not be included as they 
only had one scan. It should be noted that some of the high-risk participants had partially or fully rated psychotic symptoms at intake ( $n=12$ ), but most were working or studying productively and none had schizophrenia or any other psychotic disorder. By 1999, a relatively small number of the whole cohort $(n=7)$ had gone on to meet diagnostic criteria for schizophrenia and we are thus not yet able to examine the predictors and associations of psychosis per se. We should also stress that the current analyses were pre-planned, as were similar analyses of the neuropsychological data (Cosway et al, 2000), as part of the first phase of the study (Miller et al, 2002).

\section{Brain scanning}

Details of the image acquisition and processing are given elsewhere (Whalley et al, 1999). Briefly, participants underwent MRI scanning on a 1T Magnetom scanner (Siemens, Erlangen, Germany). Midline sagittal localisation was followed by a double spin echo sequence to identify any gross brain lesions and a fast gradient echo sequence for volumetric analysis. The latter consisted of a $180^{\circ}$ inversion pulse followed by a FLASH (fast low angle shot) collection (flip angle $12^{\circ}$, repetition/echo/inversion times $=10 / 4 / 200 \mathrm{~ms}$, relaxation delay time $500 \mathrm{~ms}$, field-of-view $250 \mathrm{~mm}$ ). This gave 128 contiguous $1.88 \mathrm{~mm}$ thick slices in the coronal plane. Semi-automated quality control checks of the signal-to-noise ratio and radio frequency field homogeneity were made daily. Inhomogeneity in the head coil was corrected for by scanning a flood phantom after each scan and normalising the data to this. Repeat scans were conducted on the same scanner, with exactly the same imaging protocol, after approximately 2 years. The scanner was not modified during the study.

Images were processed on Sun Microsystems workstations using the software package Analyze (version 7.5, Mayo Foundation, Rochester, Minnesota, USA). Seeds were placed in regions of interest, thresholds adjusted by eye and limits placed manually to separate adjoining structures. The temporal lobes and amygdala-hippocampus complex volumes were defined according to the criteria of Suddath et al (1990) and Shenton et al (1992). The temporal lobe measurements started at the temporal pole, the amygdala-hippocampus complex volume measurements were commenced when the temporal stem white matter was first observable, and the posterior boundary for both was taken as the first slice in which the crus fornicis fibres formed the medial wall of the lateral ventricle. The posterior boundary of the prefrontal lobes was taken as the first slice of the corpus callosum. Other structures were outlined by standard criteria or naturalistic boundaries as in our previous studies (Lawrie et al, 1999, 2001; Whalley et al, 1999) for exploratory analyses.

Volumetric image processing was performed by three raters (H.C.W., S.S.A., J.N.K.) with good interrater reliability: mean intraclass correlation coefficient 0.92 , range $0.78-0.99$. The main rater (H.C.W.) also measured her intrarater reliability every 6 months (mean intraclass correlation coefficient 0.94 , range 0.80 1.00). Measurement error was quantified by dividing the mean difference between a pair of ratings by the mean volume of the two. Expressed as a percentage, the mean error over time was less than $1 \%$ for the whole brain and temporal lobes, $3.2 \%$ for the amygdala-hippocampus and $1.7-5.5 \%$ for the remaining structures other than the third ventricle (Whalley et al 1999). The scans were identified by code number and date of birth only, so that the raters were masked to group membership.

\section{Statistical analysis}

All statistical testing was conducted using the Statistical Package for the Social Sciences (SPSS version 10.0 for PC). Demographics were compared between groups with analysis of variance and the chisquared test. In the high-risk group, symptom ratings on the PSE were scored as the highest symptom levels over the first 5 years of the study, dichotomised into groups of $0 / 1$ (no psychotic symptoms ever) and $2 / 3$ (psychotic symptoms). Any differential change over time in the highrisk and healthy control groups was examined with repeated measures analysis of variance (ANOVA), looking for group $x$ time interactions. The same test was employed to compare change in high-risk individuals with and without symptoms. The mean absolute change in regional volumes was calculated as the mean difference between the two scans (scan 2-scan 1 ), such that a negative value indicates volume reduction. Relative change as a percentage was calculated with reference to the appropriate volume on the first scan, i.e. $([\operatorname{scan} 2-\operatorname{scan} 1] / \operatorname{scan} 1) \times 100$.

\section{RESULTS}

\section{Changes in regional brain volumes over time}

Second scans were performed in 66 highrisk and 20 healthy participants an average of 1.8 (s.d.=0.4) years after the first assessment. The mean ages of the two groups were almost identical and there were no significant differences in the other descriptive variables (Table 1). Repeated measures ANOVA revealed no statistically significant group $\times$ time interactions. There were no significant differences in volume changes between control and high-risk participants in the left $\left(F_{1,84}=2.9, P=0.09\right)$ and right $\left(F_{1,84}=2.2, P=0.14\right)$ amygdalahippocampus, nor in the left $\left(F_{1,84}=2.3\right.$, $P=0.13)$ and right $\left(F_{1,84}=0.1, \quad P=0.7\right)$ temporal lobes. The absolute and relative changes in these and other regional brain volumes in the two groups are listed in Table 2.

\section{Changes in regional brain volumes by symptoms}

Nineteen of the high-risk participants had psychotic symptoms (12 at baseline) and

\begin{tabular}{lccc}
\hline & $\begin{array}{c}\text { High-risk group } \\
(n=66)\end{array}$ & $\begin{array}{c}\text { Control group } \\
(n=20)\end{array}$ & $P$ \\
\hline Mean age at second assessment (years) & 23.1 (s.d.=2.7) & $22.9($ s.d. $=2.2)$ & 0.8 \\
Gender (male/female) & $34 / 32$ & $13 / 7$ & 0.3 \\
Mean height (cm) & $172.3($ s.d.=10.6) & $173.0($ s.d. $=10.5)$ & 0.8 \\
Handedness (right/left/mixed) & $57 / 6 / 3$ & $16 / 2 / 2$ & 0.6 \\
Paternal social class: & & & 0.3 \\
$\quad$ Non-manual & 31 & 13 & 7 \\
$\quad$ Manual & 33 & - & \\
Unknown & 2 & & \\
\hline
\end{tabular}


Table 2 Mean absolute and relative changes in regional brain volumes in 66 high-risk and 20 control participants

\begin{tabular}{|c|c|c|c|c|}
\hline \multirow[t]{2}{*}{ Region } & \multicolumn{2}{|c|}{$\begin{array}{l}\text { Absolute change } \\
\text { (scan 2-scan I) } \\
\text { mean (s.d.) }\end{array}$} & \multicolumn{2}{|c|}{$\begin{array}{c}\text { Relative change } \\
([\text { scan } 2-\text { scan I] } / \text { scan I }) \times 100\end{array}$} \\
\hline & $\begin{array}{c}\text { High-risk } \\
\left(\mathrm{mm}^{3}\right)\end{array}$ & $\begin{array}{c}\text { Control } \\
\left(\mathrm{mm}^{3}\right)\end{array}$ & $\begin{array}{c}\text { High-risk } \\
\text { (\%) }\end{array}$ & $\begin{array}{c}\text { Control } \\
\text { (\%) }\end{array}$ \\
\hline Brain & $-2060(35590)$ & $+3152(31378)$ & -0.2 & +0.3 \\
\hline Third ventricle & $-87(158)$ & $-96(159)$ & -10.0 & -20.3 \\
\hline Fourth ventricle & $-134(217)$ & $-124(208)$ & +0.2 & -15.8 \\
\hline Left lateral ventricle & $-434(839)$ & $-365(702)$ & -7.5 & -11.0 \\
\hline Right lateral ventricle & $-399(838)$ & $-389(793)$ & -4.7 & -11.3 \\
\hline Left amygdala-hippocampus complex & $-126(445)$ & $-321(468)$ & -2.2 & -5.7 \\
\hline Right amygdala-hippocampus complex & + $+116(499)$ & $-70(464)$ & +3.5 & -1.2 \\
\hline Left temporal lobe & $-1063(4290)$ & $-2640(3044)$ & -1.2 & -3.1 \\
\hline Right temporal lobe & $-692(3373)$ & -991 (4205) & -0.8 & $-I . I$ \\
\hline Left prefrontal lobe & $-204(5217)$ & +1034 (4976) & +0.1 & +1.0 \\
\hline Right prefrontal lobe & +841 (5967) & $+1044(5967)$ & +1.6 & +1.3 \\
\hline Left thalamus & $-113(661)$ & $-348(538)$ & -0.8 & -4.7 \\
\hline Right thalamus & $-114(569)$ & $-202(465)$ & -1.2 & -3.0 \\
\hline Left caudate & $+45(285)$ & $-42(354)$ & +1.3 & -0.4 \\
\hline Right caudate & $+53(326)$ & $+6(302)$ & +1.5 & +0.3 \\
\hline Left lenticular nucleus & $+114(570)$ & $+36(43 I)$ & +2.7 & +1.2 \\
\hline Right lenticular nucleus & $+98(520)$ & $+195(473)$ & +2.3 & +4.1 \\
\hline
\end{tabular}

Table 3 Demographic characteristics of high-risk participants with and without psychotic symptoms

\begin{tabular}{lccc}
\hline & $\begin{array}{c}\text { High-risk with } \\
\text { symptoms }(n=19)\end{array}$ & $\begin{array}{c}\text { High-risk without } \\
\text { symptoms }(n=47)\end{array}$ & $P$ \\
\hline Mean age at second assessment (years) & $23.0($ s.d.=2.7) & $23.2($ s.d.=2.7) & 0.7 \\
Gender (male/female) & $7 / 12$ & $27 / 20$ & 0.1 \\
Mean height (cm) & $169.5($ s.d.=9.6) & $173.4($ s.d.=10.9) & 0.2 \\
Handedness (right/left/mixed) & $18 / 0 / 1$ & $39 / 6 / 2$ & 0.3 \\
Paternal social class: & & & 0.3 \\
$\quad$ Non-manual & 6 & 25 & 21 \\
$\quad$ Manual & 12 & 1 & \\
Unknown & 1 & & \\
\hline
\end{tabular}

47 did not. The demographic characteristics of these two groups are given in Table 3. Women and shorter individuals were non-significantly overrepresented in the high-risk group with symptoms. There was little difference in the time between scans in the two groups: $1.7($ s.d. $=0.2)$ years and 1.8 (s.d. $=0.5$ ) years, respectively.

Repeated measures ANOVA found one significant group $\times$ time interaction, in the right temporal lobe $\left(F_{1,64}=5.4, P=0.023\right)$. There was no significant interaction in the left $\left(F_{1,64}=0.2, P=0.7\right)$ or right $\left(F_{1,64}=0.7\right.$, absolute and relative changes are similar or greater in other structures such as the left and right prefrontal lobes, but the measurement error and variance are also higher.

A post hoc repeated measures ANOVA of a group $\times$ time $\times$ gender interaction for the right temporal lobe volumes in high-risk participants was not significant $(P=0.5)$. Neither of the high-risk groups significantly differed from the control group in the changes in right temporal lobe volume. The high-risk participants with psychotic symptoms at baseline and those who developed psychotic symptoms subsequently also did not differ significantly on this measure. As the numbers in the three main subject groups were unequal, we conducted paired $t$-tests for withingroup changes in temporal lobe volumes: both left and right sides showed statistically significant reductions in those with symptoms (both $P<0.015$ ) but not in those without (both $P>0.25$ ), and the brains of control subjects showed significant reductions only on the left side.

\section{DISCUSSION}

\section{Main findings}

We did not find evidence to support our first hypothesis, of a reduction in temporal lobe volumes in all high-risk individuals, as the control group showed non-significantly greater reductions. Our findings are, however, in keeping with our second hypothesis, as the right temporal lobes reduced in volume in high-risk participants with psychotic symptoms. This suggests that brain structure may change in association with psychotic symptoms.

\section{Methodological considerations}

This novel finding is unlikely to be artefactual. We tested two specific hypotheses - although we did so for bilateral structures in two different contrasts, these were not independent and would not, therefore, be suitable for a Bonferroni correction. The volume calculations were done by masked raters with good inter- and intrarater reliabilities, and measurement error was less than the main difference we found. High-risk participants with and without symptoms did not substantially differ in descriptive variables; the greatest difference was in gender distribution, but we found no symptom $x$ time $\times$ gender interaction. 
Table 4 Mean absolute and relative changes in regional brain volumes in 19 high-risk participants with psychotic symptoms and 47 high-risk participants without psychotic symptoms

\begin{tabular}{|c|c|c|c|c|}
\hline \multirow[t]{2}{*}{ Region } & \multicolumn{2}{|c|}{$\begin{array}{l}\text { Absolute change } \\
\text { (scan 2-scan I) } \\
\text { mean (s.d.) }\end{array}$} & \multicolumn{2}{|c|}{$\begin{array}{c}\text { Relative change } \\
([\text { scan } 2-\text { scan I] } / \text { scan I) } \times 100\end{array}$} \\
\hline & $\begin{array}{c}\text { Symptoms } \\
\begin{array}{c}(n=19) \\
\left(\mathrm{mm}^{3}\right)\end{array}\end{array}$ & $\begin{array}{c}\text { No symptoms } \\
\qquad \begin{array}{c}(n=47) \\
\left(\mathrm{mm}^{3}\right)\end{array}\end{array}$ & $\begin{array}{c}\text { Symptoms } \\
(n=19) \\
(\%)\end{array}$ & $\begin{array}{c}\text { No symptoms } \\
\begin{array}{c}(n=47) \\
(\%)\end{array}\end{array}$ \\
\hline Brain & $-12616(31302)$ & +2207 (36 331) & -0.9 & +0.2 \\
\hline Third ventricle & $-55(145)$ & $-100(162)$ & +6.1 & -16.5 \\
\hline Fourth ventricle & $-86(217)$ & $-154(216)$ & -9.7 & +4.2 \\
\hline Left lateral ventricle & $-428(906)$ & $-437(82 I)$ & -1.1 & -10.0 \\
\hline Right lateral ventricle & $-423(94 I)$ & $-389(803)$ & $+\mathrm{I} .4$ & -7.1 \\
\hline Left amygdala-hippocampus complex & $-87(492)$ & $-14 \mid(429)$ & -1.3 & -2.6 \\
\hline $\begin{array}{l}\text { Right amygdala-hippocampus com- } \\
\text { plex }\end{array}$ & $+32(565)$ & $+150(472)$ & +1.5 & +3.8 \\
\hline Left temporal lobe & $-1901(3050)$ & $-723(4685)$ & -2.3 & -0.8 \\
\hline Right temporal lobe & $-2163(3093)$ & $-97(3328)$ & -2.5 & -0.04 \\
\hline Left prefrontal lobe & $-334(4868)$ & $-|5|(540 \mid)$ & -0.3 & +0.3 \\
\hline Right prefrontal lobe & $-718(5845)$ & $+|47|(596 I)$ & -0.4 & +2.4 \\
\hline Left thalamus & +68 (573) & $-186(685)$ & +2.4 & -2.2 \\
\hline Right thalamus & $+74(473)$ & $-190(591)$ & +2.1 & -2.5 \\
\hline Left caudate & $-4 I(292)$ & $+79(278)$ & -0.7 & +2.1 \\
\hline Right caudate & $-68(324)$ & $+102(317)$ & -1.1 & +2.5 \\
\hline Left lenticular nucleus & $-20(634)$ & $+168(540)$ & +0.2 & +3.7 \\
\hline Right lenticular nucleus & $-65(633)$ & $+165(457)$ & -0.6 & +3.4 \\
\hline
\end{tabular}

The results do, however, need cautious interpretation. We analysed the data by symptoms rather than psychosis both to optimise statistical power and because the causes of symptom development in schizophrenia are so poorly understood. It is not therefore possible to state whether these results apply to those who will develop psychosis rather than individual psychotic symptoms, although the symptomatic participants had at least partial or isolated delusions or hallucinations and the diagnosis of schizophrenia is largely made on the grounds of these symptoms. We do not expect all the high-risk group with individual psychotic symptoms to develop frank schizophrenia, and symptoms fluctuate over time for unknown reasons. We are also not able to state whether the changes in brain structure precede or follow symptoms, as many of the symptomatic high-risk individuals had symptoms at baseline assessment. It is unlikely that the symptoms themselves effect volume reductions, but even if the temporal lobe reductions are primary, an explanation is required.
The measurement error of the volumetric technique we used is more likely to have obscured differences where they actually existed rather than to lead to false positive results. This is a possible explanation for our failure to find volume reductions in the amygdala-hippocampus in high-risk participants, particularly those with psychotic symptoms. Measurement error is in the region of $5 \%$ for these and other relatively small structures. Our previous findings of reduced amygdalahippocampus volumes at baseline could imply that those with the smallest volumes would be most likely to develop symptoms, but this is not the case - at least thus far (Lawrie et al, 2001). Alternatively, the reduced volumes of the amygdalahippocampus complex usually found in schizophrenia may only be manifested with the onset of psychosis per se.

The imaging literature on development sparse. Most brain development is

\section{Imaging brain development} changes in brain structure is relatively

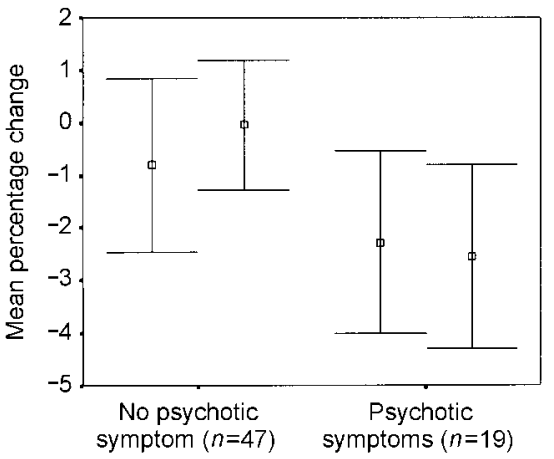

Fig. I Error bar plot (mean and $95 \%$ confidence interval) of relative (percentage) changes in the left and right temporal lobe volumes in $\mathbf{4 7}$ asymptomatic high-risk individuals and 19 individuals with psychotic symptoms.

completed in the first few postnatal months, but competitive elimination of synapses, axonal myelination and dendritic arborisation continue throughout life (Huttenlocher, 1990; Bourgeois \& Goldman-Rakic, 1994; Jernigan \& Sowell, 1997). Some MRI studies have found agerelated reductions in $T_{2}$-weighted signal suggestive of myelination throughout the hemispheres in adolescence and early adulthood (Jernigan \& Sowell, 1997). We are aware of only three MRI studies employing similar measures of regional brain volumes in control groups of similar age to our own, two from the same research group in an overlapping sample, which suggest that the frontal and temporal lobes reduce in size during early adulthood (Jernigan \& Sowell, 1997; Bartzokis et al, 2001), possibly due to 'synaptic pruning'. Automated voxel-based morphometry studies support these findings, but suggest the reductions are greater in the frontal lobes (Giedd et al, 1999; Sowell et al, 1999). Our results in healthy control participants are compatible with these findings, particularly if measurement error is taken into account. This could also explain the apparent reductions over time of ventricular volumes in the control group, but it is uncertain whether ventricular volumes begin to increase from the age of 20 years (Jernigan et al, 1991) or 30 years (Pfefferbaum et al, 1994) in healthy individuals.

These processes do not appear from our current data to differ significantly in all the high-risk participants, or in either of the high-risk groups, compared with the healthy control group. We cannot, therefore, determine whether the reductions 
in the temporal lobes in symptomatic highrisk individuals are attributable to physiological or pathological processes. The patterns of change in the temporal lobes are similar in the symptomatic high-risk and control groups, suggesting that the development of symptoms may be triggered by physiological events in the otherwise predisposed (Feinberg, 1982; Weinberger, 1987). It should be noted, however, that whole-brain volume and prefrontal lobe reductions are only apparent in the highrisk group with symptoms, which suggests that 'synaptic pruning' may be more widespread in these people but greatest in the temporal lobes. It should also be noted that post-mortem studies do not always find reductions of the amygdalahippocampus and prefrontal lobes in schizophrenia (Harrison, 1999).

\section{Clinical implications}

Structural MRI studies have reported that abnormalities of the hemispheres (DeLisi et al, 1997) and temporal lobes (Rapoport et al, 1999) may progress in the first few years after the onset of schizophrenia in patients of similar ages to our study group and at a similar rate (about $1 \%$ per year). Other studies have, however, localised changes to the frontal lobes (Gur et al, 1998) or found them to be limited to the lateral ventricles in poor-outcome subgroups (Lieberman et al, 2001). These changes could be due to antipsychotic medication effects, but none of the highrisk participants reported here was taking such medication when assessed or, indeed, at any previous time. The differences between reports may reflect differential measurement errors in semi-automated studies, and we are currently engaged in an entirely automated comparison. As far as psychotic symptoms can be localised, temporal lobe abnormalities have been linked to auditory hallucinations similar to those described by our high-risk participants (Shenton et al, 1992; Lawrie \& Abukmeil, 1998). We cannot tell from the present analysis whether particular parts of temporal lobes are reducing or whether reductions represent a general loss of temporal lobe tissue, but we are currently using more detailed techniques to determine this and any specific relationships with particular psychotic symptoms. These novel analytical methods may be sufficiently sensitive for clinical use.

\section{CLINICAL IMPLICATIONS}

Some abnormalities of brain structure in schizophrenia may precede the onset of schizophrenia.

- Reductions in temporal lobe volumes could conceivably predict the onset of schizophrenia.

These findings suggest possibilities for early detection.

\section{LIMITATIONS}

The number of high-risk participants in this study who have developed schizophrenia is, as yet, too small to determine if these findings hold in this group.

Measurement error may have obscured changes in the amygdala-hippocampus.

Temporal lobe reductions were similar in the control group.

STEPHEN M. LAWRIE, MRCPsych, HEATHER C. WHALIEY, MSc, SUHEIB S. ABUKMEIL, MRCPsych, JULIA N. KESTELMAN, BSc, PATRICK MILLER, PhD, JONATHAN J. K. BEST, FRCR, DAVID G. C. OWENS FRCPsych, EVE C. JOHNSTONE, FRCPsych, University Department of Psychiatry, Royal Edinburgh Hospital, Edinburgh, UK

Correspondence: Dr Stephen M. Lawrie, University Department of Psychiatry, Morningside Park, Edinburgh EHIO 5HF,UK. Tel: 013I 5376671 ; fax: 01315376531 ; e-mail: s.lawrie@ed.ac.uk

(First received 3 September 200I, final revision 4 April 2002, accepted 9 April 2002)

Our scanning results are compatible with our neuropsychological findings, in the same study group, of a decline in memory (and executive) function as psychotic symptoms develop (Cosway et al, 2000). It may be that the development of psychotic symptoms is triggered by one or more processes associated with reduction in temporal lobe volume and that the processes lead to the development of a psychotic illness and continuing reduction in temporal lobe volumes in at least some people. These findings therefore raise the possibility that people destined to develop schizophrenia might be identifiable before (or at least while) the disorder develops, and might potentially benefit from treatment with antipsychotic or even neuroprotective drugs.

\section{ACKNOWLEDGEMENTS}

This study was supported by the Medical Research Council of Great Britain. We thank Majella Byrne, Bobby Clafferty, Richard Cosway, Elizabeth Grant, Ann Hodges and Jane Morris for their help in acquiring the sample; Annette Blane and Ewen
Rimmington for acquiring the brain scans and correcting for inhomogeneity; general practitioners and psychiatrists throughout Scotland; and the participants and their extended families. We also thank Norma Brearley for the careful preparation of the manuscript.

\section{REFERENCES}

Bartzokis, G., Beckson, M., Lu, P. H., et al (200I) Age-related changes in frontal and temporal lobe volumes in men: a magnetic resonance imaging study. Archives of General Psychiatry, 58, 46I-465.

Bourgeois, R. P. \& Goldman-Rakic, P. S (1994) Synaptic development of the cerebral cortex: implications for learning, memory, and mental illness. Progress in Brain Resarch, 102, 227-243.

Cosway, R., Byrne, M., Clafferty, R., et al (2000) Neuropsychological change in young people at high risk for schizophrenia: results from the first two neuropsychological assessments of the Edinburgh High Risk Study. Psychological Medicine, 30, III-||2|.

DeLisi, L. E., Sakuma, M., Tew, W., et al (1997) Schizophrenia as a chronic active brain process: a study of progressive brain structural change subsequent to the onset of schizophrenia. Psychiatry Research, 74, 129-140.

Feinberg, I. (1982) Schizophrenia: caused by a fault in programmed synaptic elimination during adolescence? Journal of Psychiatric Research, 17, 319-334. 
Giedd, J. N., Blumenthal, J., Jeffries, N. O., et al (1999) Brain development during childhood and adolescence: a longitudinal MRI study. Nature Neuroscience, 2, 861-863.

Gur, R. E., Cowell, P., Turetsky, B. I., et al (1998) A follow-up magnetic resonance imaging study of schizophrenia. Relationship of neuroanatomical changes to clinical and neurobehavioural measures. Archives of General Psychiatry, 55, 145-152.

Harrison, P. J. (1999) The neuropathology of schizophrenia. A critical review of the data and their intrepretation. Brain, 122, 593-624.

Hodges, A., Byrne, M., Grant, E., et al (1999)

People at risk of schizophrenia. Sample characteristics of the first 100 cases in the Edinburgh High-Risk Study. British Journal of Psychiatry, 174, 547-553.

Huttenlocher, P. R. (1990) Morphometric study of human cerebral cortex development. Neuropsychologia, 28, 517-527.

Jernigan, T. L. \& Sowell, E. R. (1997) Magnetic resonance imaging studies of developing brain In Neurodevelopment and Adult Psychopathology (eds M. S. Keshavan \& R. M. Murray), pp. 63-70. Cambridge: Cambridge University Press.

_ , Trauner, D. A., Hesselink, J. R., et al (199I)

Maturation of human cerebrum observed in vivo during adolescence. Brain, II4, 2037-2049.

Johnstone, E. C., Abukmeil, S. S., Byrne, M., et al (2000) Edinburgh high risk study - findings after four years. Demographic, attainment and psychopathological issues. Schizophrenia Research, 46, I-15.

Lawrie, S. M. \& Abukmeil, S. S. (1998) Brain abnormality in schizophrenia. A systematic and quantitative review of volumetric magnetic resonance imaging studies. British Journal of Psychiatry, I72, II0-120.

_ , Whalley, H., Kestelman, J. N., et al (1999) Magnetic resonance imaging of brain in people at high risk of developing schizophrenia. Lancet, 353. 30-33.

_ , _ , Abukmeil, S. S., et al (200I) Brain structure genetic liability and psychotic symptoms in subjects at high risk of developing schizophrenia. Biological Psychiatry, 49, 81I-823.

Lieberman, J., Chakos, M., Wu, H., et al (200I)

Longitudinal study of brain morphology in first episode schizophrenia. Biological Psychiatry, 49, 487-499.

McGuffin, P., Farmer, A. \& Harvey, I. (1991) A polydiagnostic application of operational criteria in studies of psychotic illness. Development and reliability of the OPCRIT system. Archives of General Psychiatry, 48, 764-770.

Miller, P. M., Byrne, M., Hodges, A., et al (2002) Childhood behaviour, psychotic symptoms and psychosis onset in young people at high risk of schizophrenia: early findings from the Edinburgh high risk study. Psychological Medicine, 32, 173-179.

Pfefferbaum, A., Mathalon, D. H., Sullivan, E.V., et al (1994) A quantitative magnetic resonance imaging study of changes in brain morphology from infancy to late adulthood. Archives of Neurology, 5I, 874-887.

Rapoport, J. L., Giedd, J. N., Blumenthal, J., et al (1999) Progressive cortical change during adolescence in childhood-onset schizophrenia. A longitudinal magnetic resonance imaging study. Archives of General Psychiatry, 56, 649-654
Shenton, M. E., Kikinis, R., Jolesz, F. A., et al (1992) Abnormalities of the left temporal lobe and thought disorder in schizophrenia. A quantitative magnetic resonance imaging study. New England Journal of Medicine, 327, 604-612.

Sowell, E. R., Thompson, P. M., Holmes, C. J., et al (1999) In vivo evidence for post-adolescent brain maturation in frontal and striatal regions. Nature Neuroscience, 2, 859-861.

Suddath, R. L., Christison, G.W., Torrey, E. F., et a (1990) Anatomical abnormalities in the brains of monozygotic twins discordant for schizophrenia. New England Journal of Medicine, 322, 789-794.

Weinberger, D. R. (1987) Implications of normal brain development for the pathogenesis of schizophrenia. Archives of General Psychiatry, 44, 660-669.

Whalley, H. C., Kestelman, J. N., Rimmington, J. E., et al (1999) Methodological issues in volumetric magnetic resonance imaging of the brain in the Edinburgh High Risk Project. Psychiatry Research, 91, 31-44.

Wing, J. K., Cooper, J. E. \& Sartorius, N. (1974) The Measurement and Classification of Psychiatric Symptoms. Cambridge: Cambridge University Press.

World Health Organization (1992) Tenth Revision of the International Classification of Diseases and Related Health Problems (ICD-I0). Geneva: WHO.

Wright, I. C., Rabe-Hesketh, S., Woodruff, P. W., et al (2000) Meta-analysis of regional brain volumes in schizophrenia. American Journal of Psychiatry, 157 16-25. 